\title{
Avant-propos
}

\section{Le dossier médical et le patient à besoins spécifiques}
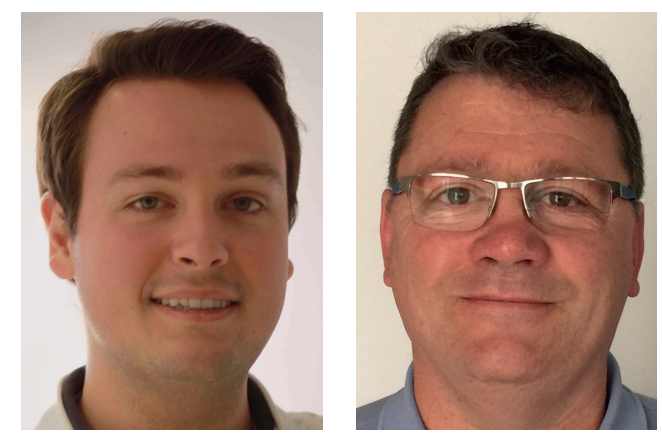

D. Brézulier ${ }^{1}$, O. Sorel ${ }^{2}$

1 Assistant Hospitalier Universitaire, Spécialiste Qualifié, CHU Rennes, Université de Rennes 1

2 Professeur des Universités - Praticien Hospitalier, Spécialiste Qualifié, CHU Rennes, Université de Rennes 1

Le philosophe Mark Hunyadi de I'Université Laval définit trois notions complémentaires et incontournables en Science, qui plus est en Médecine :

- la prudence vise les risques avérés dont la fréquence d'apparition est connue ;

- la prévention, quant à elle, comble les risques avérés dont la fréquence n'est pas clairement définie ;

- enfin la précaution s'applique aux risques dont ni I'ampleur ni la probabilité d'occurrence ne peuvent être calculées avec certitude.

Prudence et précaution peuvent être envisagées comme des freins à l'innovation et au progrès des thérapeutiques. D'ailleurs ne parle-t-on pas de principe de précaution?

Ce principe, appliqué aux soins orthodontiques, peut également se concevoir en termes de niveau de connaissances, d'exigences... dans la finalité de rendre nos prises en charge plus sûres, plus efficientes.
On prend alors pleinement conscience du poids « de la connaissance » dans le rapport bénéfice/risque.

Toutefois, "la connaissance » ne peut plus se cantonner au pur domaine technique. C'est en partant de ce postulat que nous avons choisi de consacrer un numéro de la Revue d'Orthopédie Dento-Faciale aux patients à besoins spécifiques, heureusement peu nombreux et par conséquent dont nous n'avons que peu l'habitude, mais requérant toute notre vigilance.

Dans ce numéro, Yves Soyer propose de dresser le cadre médico-légal du dossier médical du patient, élément ô combien important et souvent premier contact avec les particularités des patients à besoins spécifiques.

Un filet de Bernard Peckels nous fera réfléchir sur ce qu'est «l'état de bonne santé ».

Nous commencerons ensuite la prise en charge de nos patients à besoins spécifiques, tout d'abord ceux 
suivis en oncologie pédiatrique avec l'article d'Émile Boyer.

Puis nous évoquerons le cas des patients atteints d'endocrinopathies et verrons quelles sont les répercussions de ces atteintes sur la croissance et la maturation squelettique et dentaire grâce à l'article de Fanny Duhamel.

Nous envisagerons ensuite la prise en charge des patients souffrant de cardiopathies avec le travail d'Olivier Leborgne.

La prise en charge interceptive des patients atteints du syndrome de Down est traitée par Jean-Louis Sixou.
Enfin, nous nous attarderons grâce au travail de Mickael Sebbag et de l'équipe bordelaise sur le diagnostic et la prise en charge des patients souffrant du syndrome de Brodie.

Nous conclurons ce numéro par un cas clinique traité par Bertrand Ducroz au sein de l'unité fonctionnelle d'ODF du CHU de Rennes.

Nous vous souhaitons à toutes et à tous une très bonne lecture, en espérant que ce numéro réponde à vos questions. 